\title{
Color Image Contrast Enhancement using Daubechies D4 Wavelet and Luminance Analysis
}

\author{
Murtaza Saadique Basha \\ Research Scholar, Department of Computer \\ Science \& Engineering, Manonmaniam Sundaranar \\ University, Tirunelveli, Tamil Nadu, India
}

\author{
M.Ramakrishnan \\ Professor \& Head, Department of Information \\ Technology, Velammal Engineering College, \\ Chennai-66, Tamil Nadu, India
}

\begin{abstract}
This paper presents a novel approach which address contrast enhancement in color images. The wavelet transform decomposes an image into bands that vary in spatial frequency and orientation. The HSV color model is well suited for color image enhancement methods even though RGB color space is the predominant one. By using Daubechies D4 wavelet transformation and HSV color model, a novel method of color image enhancement based on luminance adjustment is proposed here. The proposed method not only enables approximating digital signals in a better way but also it approximates highly non-linear digital signals. The experimental results showed that this new method can enhance color images effectively.
\end{abstract}

\section{General Terms}

Colour Image Enhancement Daubechies Wavelet Transform HIS Analysis Histogram Equalization luminance enhancement, HSV color space, wavelet transform

\section{Keywords}

Colour Image Enhancement Daubechies Wavelet Transform HIS Analysis Histogram Equalization luminance enhancement, HSV color space, wavelet transform

\section{INTRODUCTION}

There are various sources of image capturing. These images often contain noise leading to poor visualization. Another one difficulty with noisy images is that they are not suitable for subsequent processing. So these images need enhancement. The purpose of image enhancement is to improve images in terms of visual appearance for human interpretation on making it suitable for further computer processing[1]. One or more characteristic features of an image can mutate while performing image enhancement process [1][2].

Image enhancement is one of the basic requirements for making an image useful for various statistical analyzes. There are various techniques exist for enhancing an image and these techniques are broadly classified into two major categories[3]:

\section{1) Spatial domain}

2) Frequency domain

In spatial domain approach, the pixels of an image are altered directly. Frequency domain approach operates on Fourier transform of an image and it is then transformed back to the spatial domain[3]. In the spatial domain method, the pixel comprising of image details are considered and various procedures are directly applied on these pixels ie. $\mathrm{g}(\mathrm{x}, \mathrm{y})=$ $T[f(x, y)]$ where $f(x, y)$ is the input image, $g(x, y)$ is the output image and $T$ represents an operation on ' $f$ ' defined over some neighborhood of $(\mathrm{x}, \mathrm{y})$.

The convolution theorem is the basis for the frequency domain approaches. Let $g(x, y)$ be an image formed by the convolution of the image $f(x, y)$ and linear position invariant operator $\mathrm{h}(\mathrm{x}, \mathrm{y})$ and it is given by

$$
\mathrm{G}(\mathrm{x}, \mathrm{y})=\mathrm{H}(\mathrm{x}, \mathrm{y}) * \mathrm{f}(\mathrm{x}, \mathrm{y}),
$$

where '*' represents the convolution operator. In a typical image enhancement application, the above equation is modified as

$$
\mathrm{g}(\mathrm{x}, \mathrm{y})=\mathrm{F}-1[\mathrm{H}(\mathrm{u}, \mathrm{v}) \mathrm{F}(\mathrm{u}, \mathrm{v})]
$$

The edges in $\mathrm{f}(\mathrm{x}, \mathrm{y})$ can be highlighted using a function $\mathrm{H}(\mathrm{u}, \mathrm{v})$ that emphasize the high frequency components of $F(u, v)$.

Both spatial domain techniques and frequency domain techniques are used for color image enhancement. The spatial domain techniques are simple and easy to implement and also operation speed is very high. Contrast stretching, histogram equalization, thresholding, mask processing (or) filtering, etc are some of the spatial domain techniques[3]. Inspite of its advantages, there are certain situations in which spatial filters are not easily addressable and in these situations the best option is frequency domain techniques.

As said earlier, frequency domain technique uses Fourier transform for image enhancement. There are several transforms available such as Fourier transform, Hilbert transform, Wavelet transform, etc. The wavelet transform is better than Fourier transform because it gives frequency representation of a raw signal at any given interval of time.

Buzuloiu et al proposed a image color image scheme based on adaptive neighborhood histogram equalization[4]. The main idea of this approach is to use two levels of equalization viz. global and local. The new intensity of a pixel is calculated based on global histogram equalization function with respect to the pixel values of neighborhood[4].

Shyu et al[5] suggested a genetic algorithm in which enhancement problem is formulated as an optimization problem. Recent image enhancement techniques are based on multiscale technologies. $\mathrm{Lu}[6]$ proposed a contrast enhancement method based on multi scale gradient transformation.

Sakellaropoulos et al[7] proposed an image enhancement method using wavelet based spatially adaptive method. Chung - Liang et al[8] proposed image enhancement method by adjusting hue, saturation and intensity of the image. It describes which are the essential components that need enhancement needed for the whole image enhancement.

The literature survey described above is enough for our proposed color image enhancement method. In our proposed method, color shifting has been considered. Care has been taken so that our image enhancement method should not convert, for example, a red color to a yellow color. The range of colors that are physically obtainable by a color system is called the gamut of the device. Since we are converting the 
image from one color space to another, it may so happen that our method will have an effect of gamut problem. Care has been taken that our image enhancement avoids gamut problem.

This paper is organized as follows. Section 2 describes the methodology and our approach for feature enhancement. Section 3 shows basic color space conversion, luminance contrast enhancement and our proposed method. Section 4 describes the experimental results and section 5 describes the conclusion and future enhancement.

\section{METHODOLOGY}

\subsection{Color Space}

Color space, also known as color model (color system) is a mathematical abstract model which describes the range of colors as tuples of numbers. A range of colors can be created by the primary colors of pigment and these colors then define a specific color space. There are a variety of color spaces such as RGB, CMYK, HSV, etc.

RGB is a kind of color space which uses red, green and blue to represent a color. These three colors are considered to be the primary colors of the visible light spectrum. In this color model, each pixel of an image is assigned a range of 0 to 255 intensity values of RGB components. Using only these three colors, there can be $16,777,216$ colors on the screen by different mixing ratios.

RGB is a additive and subtractive model, defining color in terms of the combinations of primaries, where as HSV color space encapsulates information about a color in terms that are more familiar to humans[10]. HSV color space is quite similar to the way in which humans perceive a color. The colors used in HSV can be clearly defined by human perception, which is not always the care with RGB and CMYK[10]. Therefore HSV color model is best suited for color image enhancement.

The HSV color space contains three components; hue, saturation and luminance. Hue is the attribute of a color, which decides which color it is. It is that quality of the color by which we distinguish one color family from another. For color image enhancement, it is necessary that hue value should not get changed for any pixel[10]. If hue gets changed, then original color of the image gets changed thereby distorting the image[10].

Saturation is an expression for the relative bandwidth of the visible output from a light source. It indicates the range of grey in the color space. It ranges from 0 to $100 \%$. Sometimes it may range from 0 to 1 . A pure red is fully saturated, with a saturation of 1 ; tints of red have saturation less than 1 and white has a saturation of 0 .

Chrominance describes the color channels in an image, ranging from the absence of color to the maximum levels of a color. Chrominance value can be described using two properties; hue and saturation. Since these two are almost identical, we keep these two unchanged in our image enhancement method.

\subsection{Luminance Enhancement}

As said earlier, we will apply transformation and reverse transformation only to the luminance component. The luminance value describes how dark the color is. A value of 0 is black, with increasing lightness moving away from black.

Wavelet means small waves and the study of wavelet transform is a new tool for seismic signal analysis. In conventional Fourier transform, we use sinusoids for basis functions. It can only provide the frequency information. Temporal information is lost in this transformation process. It can be shown that we can have both frequency and temporal information by this kind of transform using wavelets.

Images are basically matrices. For this reason, image processing can be regarded as matrix processing. It is the fact that human vision is much more sensitive to small variations in color or brightness ie. human vision is more sensitive to low frequency signals. Therefore, high frequency components in images can be compressed without distortion. Wavelet transform is one of the best tool for us to determine where the low frequency area and high frequency area is.

$\mathrm{Xu}$ et al [11] developed a noise filtration method based on the spatial correlation between wavelet coefficients over adjacent scales. Wavelet can decompose a signal to two parts; a low frequency part, which is some sort of average of the original signal and high frequency part, which is remaining after the low frequency part is subtracted from the original signal. In our approaches, we exploit this characteristic feature of wavelet transform and we call these two frequency parts as approximate components and detail components. Approximate components are enhanced by increasing the contrast.

According to human visual theory, retina contains two types of vision cells or photo receptors called rods and cones. Rods are long, cylindrical cells that allow for night vision and sight of general shapes and forms. The cones are responsible for day time vision, color vision and the finer details of the things we look at. Kobayashi et al[12] proposed a reverse S-shape transform to enhance the grey-level image after analyzing the features of human visual system. This concept is applied to transform the luminance component of the color image.

\section{ALGORITHM OVERVIEW}

\subsection{Color Model Conversion}

As described earlier, we apply our enhancement method in HSV color space. In general color images are represented in RGB color space and therefore it is necessary to convert image from RGB color model to HSV color model.

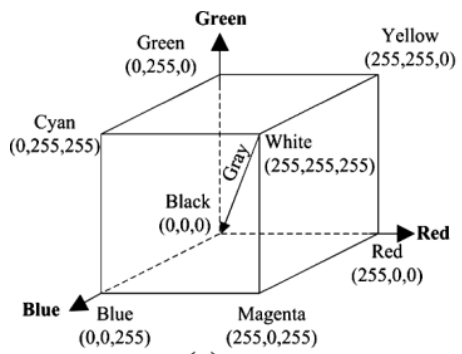

(a)

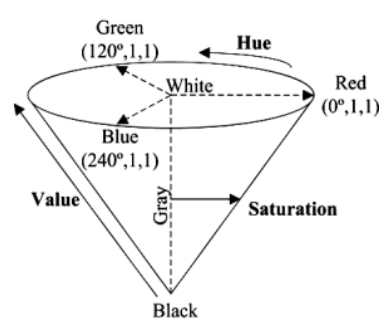

(b)
The conversion formula is shown in the below equation. Max is the maximum value in R,G,B and min is the minimum value in $R, G, B$

$$
\begin{aligned}
& \max =\operatorname{MAX}(\mathrm{R}, \mathrm{G}, \mathrm{B}) \\
& \min =\operatorname{MIN}(\mathrm{R}, \mathrm{G}, \mathrm{B})
\end{aligned}
$$




$$
\begin{aligned}
& h=\left\{\begin{array}{lr}
0 & \text { if } \max =\min \\
\left(60^{\circ} \times \frac{g-b}{\max -\min }+0^{\circ}\right) \bmod 360^{\circ} & \text { if } \max =\mathrm{r} \\
60^{\circ} \times \frac{b-r}{\max -\min }+120^{\circ} & \text { if } \max =\mathrm{g} \\
60^{\circ} \times \frac{r-g}{\max -\min }+240^{\circ} & \text { if } \max =\mathrm{b}
\end{array}\right. \\
& s=\left(\begin{array}{lr}
0 & \text { if } \max =0 \\
\frac{\max -\min }{\max }=1-\frac{\min }{\max }, & \text { otherwise }
\end{array}\right. \\
& v=\max
\end{aligned}
$$

After performing enhancement in HSV color space, we need to convert the enhanced image back to RGB color space to display/store the result. Converting HSV to RGB can be accomplished by using the below equation

$$
\begin{aligned}
& \begin{array}{l}
c=v * s \\
m=v-c
\end{array} \\
& x=c *\left(\left|\frac{h}{60} \bmod 2-1\right|\right) \\
& (R, G, B)= \begin{cases}(c+m, x+m, m) & \text { if } \mathrm{h} \in[0,60] \\
(x+m, c+m, m) & \text { if } \mathrm{h} \in[60,120] \\
(m, c+m, x+m) & \text { if } \mathrm{h} \in[120,180] \\
(m, x+m, c+m) & \text { if } \mathrm{h} \in[180,240] \\
(x+m, m, c+m) & \text { if } \mathrm{h} \in[240,300] \\
(c+m, m, x+m) & \text { if } \mathrm{h} \in[300,360]\end{cases}
\end{aligned}
$$

\subsection{Contrast Enhancement of Approximate Components}

To enhance luminance component $(\mathrm{V})$, the first step is to distinguish the luminance into two components viz. approximate components and detail components. This can be done by using wavelet transformation. We apply contrast enhancement technique to the approximate components and then reconstruct the brightness by applying inverse wavelet transformation. Thus it contains three procedures:-
i) Wavelet transformation
ii) Contrast enhancement
iii) Inverse wavelet transformation

Wavelet transformation for one dimensional signal $x(t)$ is given by the equation

$$
W_{f}(a, b)=\frac{1}{\sqrt{|a|}} \int_{-x}^{x} x(t) \psi *\left(\frac{a-b}{a}\right) d t
$$

The continuous wavelet transform is a function of two variable $a$ and $b$. Here ' $a$ ' is the scaling parameter and ' $b$ ' is the shift parameter. $\psi(x)$ is the mother wavelet or the basis function and it is used as a prototype for generating all the basis function.

Based on orthogonal wavelet transform, the luminance value is decomposed by using the equation

$$
F(x, y)=\sum_{j=0}^{n-1} A_{j} \phi_{j n}(x, y)+\sum_{j=0}^{n-1} \sum_{k=0}^{n} D_{j k} \psi(x, y)
$$

where $\phi$ is the scaling function and $\psi$ is the wavelet function. The first one represents the approximate components and the later one represents the details components

$$
\begin{aligned}
& A_{j} \text {-- approximate components } \\
& D_{j k} \text {-- detail components }
\end{aligned}
$$

The value of $A_{j}$ is in the range $[0,255]$ as $A_{j}$ represents luminance value. Not all the values of $A_{j}$ are enhanced as this will lead to image distortion. In order to overcome this problem, two threshold values are introduced in our method $\mathrm{m}$ and $\mathrm{M}$ such that whole range of $A_{j}$ is divided in to three parts $[0, \mathrm{~m}][\mathrm{m}, \mathrm{M}][\mathrm{M}, 255]$. If the value of $A_{j}<\mathrm{m}$ or $A_{j}$ $>\mathrm{M}$, we do not perform any operation on this value as this will distort the image. Otherwise we use the transform model represented by the equation

$$
R(I)=\log \frac{I-K_{1}}{K_{2}-I}
$$

I indicates the input and $\mathrm{R}$ indicates the output result. Parameters $\mathrm{K}_{1}$ should be less than the minimum of input $\mathrm{I}$ and $\mathrm{K}_{2}$ should be larger than the maximum of input $\mathrm{I}$.

\subsection{Steps}

- Compute $\mathrm{R}$ for each $A_{j}$ within the range [m, M]

- $\quad$ Normalize $\mathrm{R}$ by

$$
R^{\prime}=\frac{R-R_{\min }}{R_{\max }-R_{\min }}
$$

where Rmax and Rmin are the maximum and minimum values of all the $\mathrm{R}$ obtained using the equation (5)

- Get the new values of $A_{j}$

$$
A^{\prime}=R^{\prime} *(M-m)+m
$$

Te image is reconstructed by using the inverse wavelet transformation given by the equation

$$
F^{\prime}(x, y)=\sum_{j=0}^{n-1} A_{j}^{\prime} \phi_{j n}(x, y)+\sum_{j=0}^{n-1} \sum_{k=0}^{n} D_{j k} \psi(x, y)
$$

The next problem to be addressed is assigning the threshold 
values $\mathrm{m}$ and $\mathrm{M}$. There are two ways by which this can be done. One way is manual assignment and another way is automatic assignment. Former one is very simple and it assigns a value to the threshold parameters. But if most of the coefficients are near the maximum value, we need to set a larger threshold otherwise most coefficients are not changed and resulting picture will not have good enhancement. On the other hand, if most of the values are minimal, the same situation can occur. To solve this difficulty, we use automatic assignment. In this type, threshold value is calculated according to the information of the input image. Computation of threshold value is as follows:

- If most of the $A_{j}$ values are between m and $\mathrm{M}$, the coefficients that are out of range $[\mathrm{m}, \mathrm{M}]$ are not converted.

- The range $[\mathrm{m}, \mathrm{M}]$ should not be too small. Otherwise, the effect of the enhancement is not significant, because the results are also in $[\mathrm{m}, \mathrm{M}]$

The algorithm includes the following three steps

i) Assign two constants $\mathrm{m} 0$ and $\mathrm{M} 0$ with the conditions $\mathrm{m} 0<\mathrm{M} 0<=255$ and $\mathrm{M} 0-\mathrm{m} 0>150$

ii) Compute the values of $\mathrm{m} 1$ and $\mathrm{M} 1$ such that only $5 \%$ of approximate coefficients $\left(A_{j}\right)$ are less than $\mathrm{m} 1$ and 5\% are greater than M1. (By doing this our algorithm can change $90 \%$ of the $A_{j}$ coefficients)

iii) Set $\mathrm{m}=\min (\mathrm{m} 0, \mathrm{~m} 1)$ and $\mathrm{M}=\mathrm{Max}(\mathrm{M} 0, \mathrm{M} 1)$

\subsection{Saturation Enhancement}

According to the relationship between intensity and saturation, the maximum saturation value is calculated as follows:

$$
S_{\max }= \begin{cases}2\left(\frac{1}{I}-1\right) \frac{2}{3}<I \leq 1 \\ 1 & 0<\mathrm{I} \leq \frac{2}{3}\end{cases}
$$

The pixels with $\mathrm{I} \leq \frac{2}{3}$, there is no need to correct saturation value. But for pixels with $\mathrm{I}>\frac{2}{3}$, the correction of saturation after enhancement is a necessary procedure. If the saturation after enhancement exceeds $S_{\max }$, it must be set to $S_{\max }$ to avoid possible hue distortion after HSV to RGB transformation. Strickland et al[13] has been shown that saturation component often contains image details than luminance. In our research, we use histogram equalization.

Our method works as follows

1) Input a color image

2) If the input image is not in HSV color space, convert it to HSV color space.

3) Apply the wavelet transformation to luminance component and decompose it to approximate components and detail components.

4) By using threshold values, enhance luminance component
5) Reconstruct the image using inverse wavelet transform

6) Apply saturation enhancement for pixels has crossed Smax value.

7) Convert the image from HSV to RGB color space

8) Store the image

\section{EXPERIMENTAL RESULTS}

We have applied our method to a low contrast image and compared the results with histogram equalization. Figure (1) shows the original image, Figure (2) histogram of the original image, Figure(3) Image Enhanced by Histogram Equalization, Figure(4) Image enhanced by our method and Figure(5) Histogram after enhancement by our method.

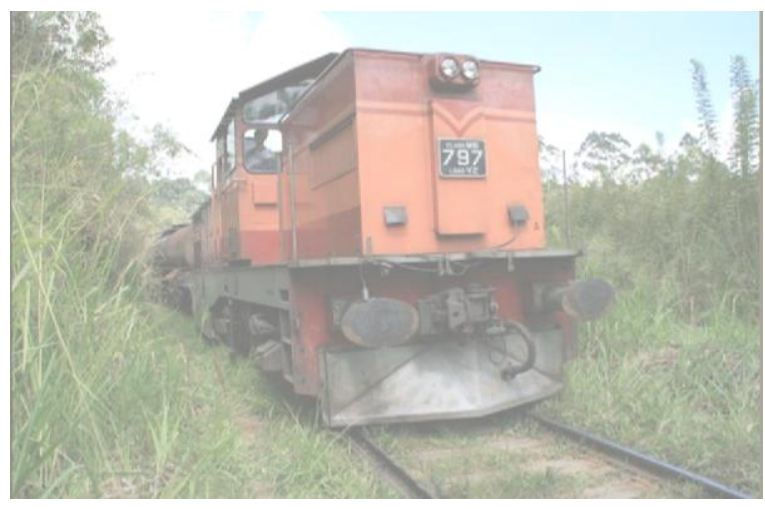

Figure(1) - original Image

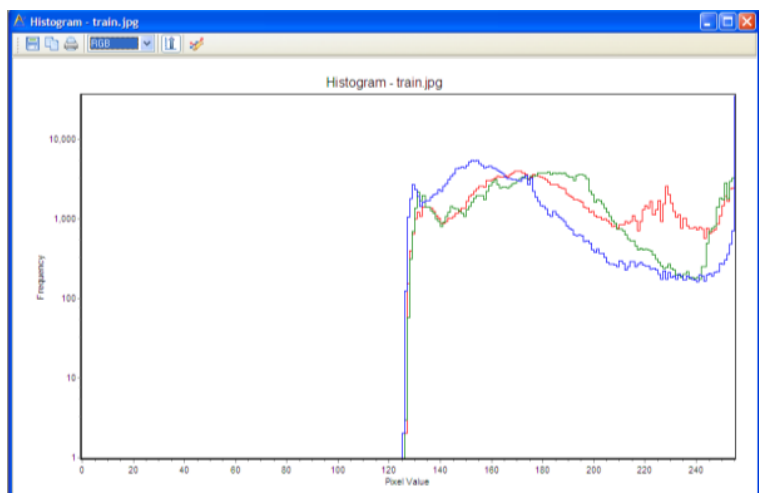

Figure (2) - Histogram of the original Image

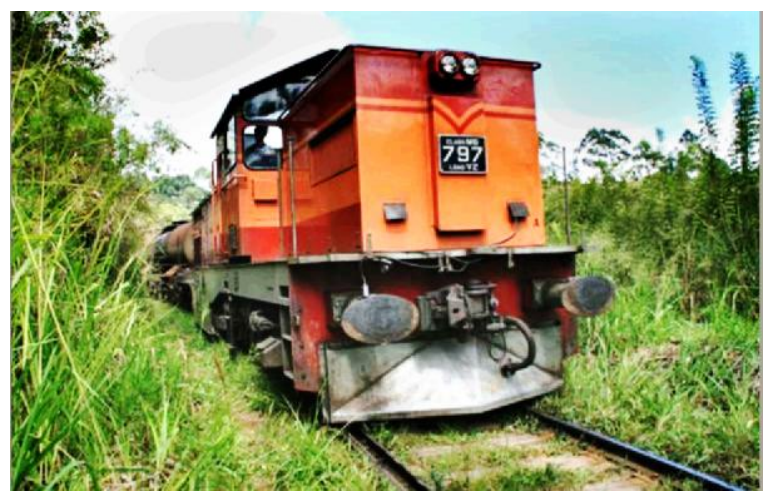

Figure (3) - Image enhanced using Histogram Equalization 


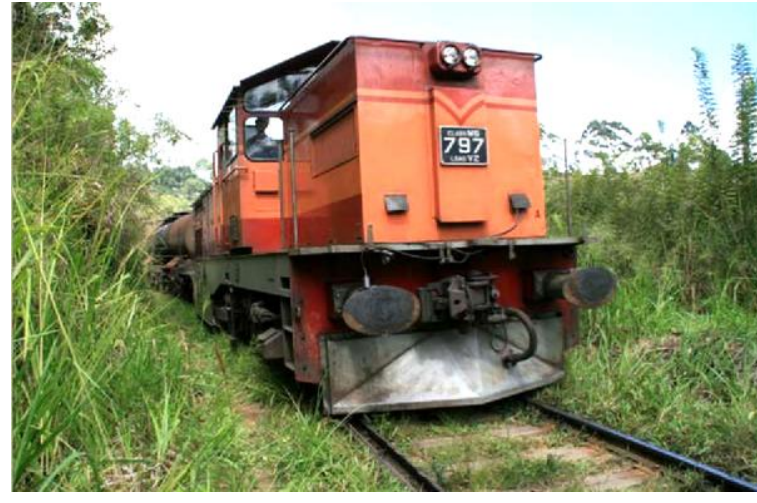

Figure (4) - Image Enhancement using our method

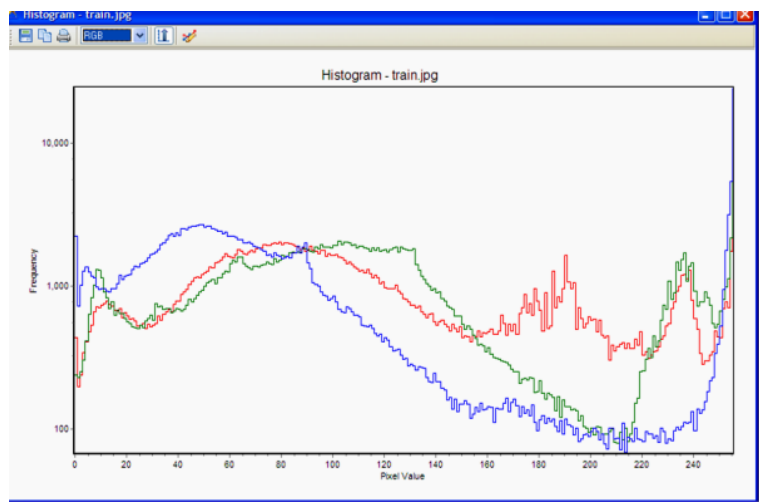

Figure(5) - Histogram - after enhancement by our method

Experimental results are shown in the above figures. Histogram equalization is a statistical method and cannot work well when there are high intensity distributions in the image. Wavelet based methods are usually artifact free and because of denoising capability of wavelet transform, are mostly used for contrast enhancement in noisy environments. Comparing histogram equalization and our proposed method, experimental results shows that our method can successfully enhance the image in an effective way.

\section{CONCLUSION}

In this paper, we have presented an approach for color image enhancement by Daubechies D4wavelet transform and HIS analysis. The luminance component is decomposed and approximate coefficients are enhanced based on threshold values. Saturation is slightly adjusted to make the enhanced image soft and vivid. The proposed approach is more understandable and flexible. However, this method is still dependent on threshold value assignment.

\section{REFERENCES}

[1] Raman Maini and Himanshu Aggarwal, "A Comprehensive Review of Image Enhancement Techniques" JOURNAL OF COMPUTING, VOLUME 2, ISSUE 3, MARCH 2010

[2] Bhabatosh Chanda and Dwijest Dutta Majumder, 2002, Digital Image Processing and Analysis.

[3] M. Ramakrishnan and Murtaza Saadique Basha "Color Image Enhancement based on Daubechies Wavelet and HIS Analysis" International Journal of Computer Applications Volume 47 - Number 13, 2012

[4] Vasile Buzuloiu, Mihai Ciuc, Rangaraj M. Rangayyan and Constantin Vertan " Adaptive-Neighborhood Histogram Equalization of Color Images" Journal of Electronic Imaging March 2000

[5] M. S. Shyu, \& J. J. Leou, A genetic algorithm approach to color image enhancement, International Journal of Pattern Recognition, 31(7), 1998, 871-880.

[6] J. Lu, \& D. M. Hearly, Contrast enhancement via multiscale gradient transformation, Proc. SPIE Conf. on Wavelet Application Orlando, FL,USA, 1994, 345-365.

[7] P Sakellaropoulos, L Costaridou and G Panayiotakis “A wavelet-based spatially adaptive method for mammographic contrast enhancement"

[8] B. A. Thomas, R. N. Strickland, \& J. J. Rodriguez, Color image enhancement using spatially adaptive saturation feedback, Proc. $4^{\text {th }}$ IEEE Conf. on Image Processing, Santa Barbara, CA, USA, 1997, 30-33.

[9] Sarif Kumar Naik and C. A. Murthy "Hue-Preserving Color Image Enhancement Without Gamut Problem" IEEE TRANSACTIONS ON IMAGE PROCESSING, VOL. 12, NO. 12, DECEMBER 2003

[10] R. S. Ledley, M. Buas, \& T. J. Colab, Fundamentals of true-color image processing, Proc. $10^{\text {th }}$ IEEE Conf. on Pattern Recognition, Los Alamos, CA, USA, 1990.

[11] Y. Xu, J. B. Weaver, D. M. Healy, \& J. Lu, Wavelet transform domain filters: A spatially selective noise filtration technique, IEEE Trans. on Image Processing, 3(6), 1994, 747-758

[12] Y. Kobayashi, \& T. Kato, A high fidelity contrast improving model based on human vision mechanism, Proc. IEEE International Conf. on Multimedia Computing and Systems, Florence, Italy, 1999, 578-584.

[13] R. N. Strickland, C. S. Kim, \& W. F. McDonnell, Digital color image enhancement based on the saturation component, International Journal of Optical Engineering, 26(7),1987,609-616 